\title{
The Case for a Single-Chip Multiprocessor
}

\author{
Kunle Olukotun, Basem A. Nayfeh, Lance Hammond, Ken Wilson, and Kunyung Chang
}

\author{
Computer Systems Laboratory \\ Stanford University \\ Stanford, CA $94305-4070$ \\ http://www-hydra.stanford.edu
}

\begin{abstract}
Advances in IC processing allow for more microprocessor design options. The increasing gate density and cost of wires in advanced integrated circuit technologies require that we look for new ways to use their capabilities effectively. This paper shows that in advanced technologies it is possible to implement a single-chip multiprocessor in the same area as a wide issue superscalar processor. We find that for applications with little parallelism the performance of the two microarchitectures is comparable. For applications with large amounts of parallelism at both the fine and coarse grained levels, the multiprocessor microarchitecture outperforms the superscalar architecture by a significant margin. Single-chip multiprocessor architectures have the advantage in that they offer localized implementation of a high-clock rate processor for inherently sequential applications and low latency interprocessor communication for parallel applications.
\end{abstract}

\section{Introduction}

Advances in integrated circuit technology have fueled microprocessor performance growth for the last fifteen years. Each increase in integration density allows for higher clock rates and offers new opportunities for microarchitectural innovation. Both of these are required to maintain microprocessor performance growth. Microarchitectural innovations employed by recent microprocessors include multiple instruction issue, dynamic scheduling, speculative execution and non-blocking caches. In the future, the trend seems to be towards CPUs with wider instruction issue and support for larger amounts of speculative execution. In this paper, we argue against this trend. We show that, due to fundamental circuit limitations and limited amounts of instruction level parallelism, the superscalar execution model will provide diminishing returns in performance for increasing issue width. Faced with this situation, building a complex wide issue superscalar CPU is not the most efficient use of silicon resources. We present the case that a better use of silicon area is a multiprocessor microarchitecture constructed from simpler processors.

Permission to make digital/hard copy of part or all of this work for personal or classroom use is granted without fee provided that copies are not made or classroom use is granted without fee proviage, the copyright notice, the title of the publication and its date appear, and notice is given that copying is by permission of ACM, Inc. To copy otherwise, to republish, to post on servers, or to redistribute to lists, requires prior specific permission and/or a fee.

ASPLOS VII 10/96 MA, USA

(1) 1996 ACM 0-89791-767-7/96/0010...\$3.50
To understand the performance trade-offs between wide-issue processors and multiprocessors in a more quantitative way, we compare the performance of a six-issue dynamically scheduled superscalar processor with a $4 \times$ two-issue multiprocessor. Our comparison has a number of unique features. First, we accurately account for and justify the latencies, especially the cache hit time, associated with the two microarchitectures. Second, we develop floor-plans and carefully allocate resources to the two microarchitectures so that they require an equal amount of die area. Third, we evaluate these architectures with a variety of integer, floating point and multiprogramming applications running in a realistic operating system environment.

The results show that on applications that cannot be parallelized, the superscalar microarchitecture performs $30 \%$ better than one processor of the multiprocessor architecture. On applications with fine grained thread-level parallelism the multiprocessor microarchitecture can exploit this parallelism so that the superscalar microarchitecture is at most $10 \%$ better. On applications with large grained thread-level parallelism and multiprogramming workloads the multiprocessor microarchitecture performs $50-100 \%$ better than the wide superscalar microarchitecture.

The remainder of this paper is organized as follows. In Section 2, we discuss the performance limits of superscalar design from a technology and implementation perspective. In Section 3, we make the case for a single chip multiprocessor from an applications perspective. In Section 4, we develop floor plans for a six-issue superscalar microarchitecture and a $4 \times$ two-issue multiprocessor and examine their area requirements. We describe the simulation methodology used to compare these two microarchitectures in Section 5, and in Section 6 we present the results of our performance comparison. Finally, we conclude in Section 7.

\section{The Limits of the Superscalar Approach}

A recent trend in the microprocessor industry has been the design of CPUs with multiple instruction issue and the ability to execute instructions out of program order. This ability, called dynamic scheduling, first appeared in the CDC 6600 [21]. Dynamic scheduling uses hardware to track register dependencies between instructions; an instruction is executed, possibly out of program order, as soon as all of its dependencies are satisfied. In the CDC 6600 the register dependency checking was done with a hardware structure called the scoreboard. The IBM 360/91 used register renaming to improve the efficiency of dynamic scheduling using hardware struc- 


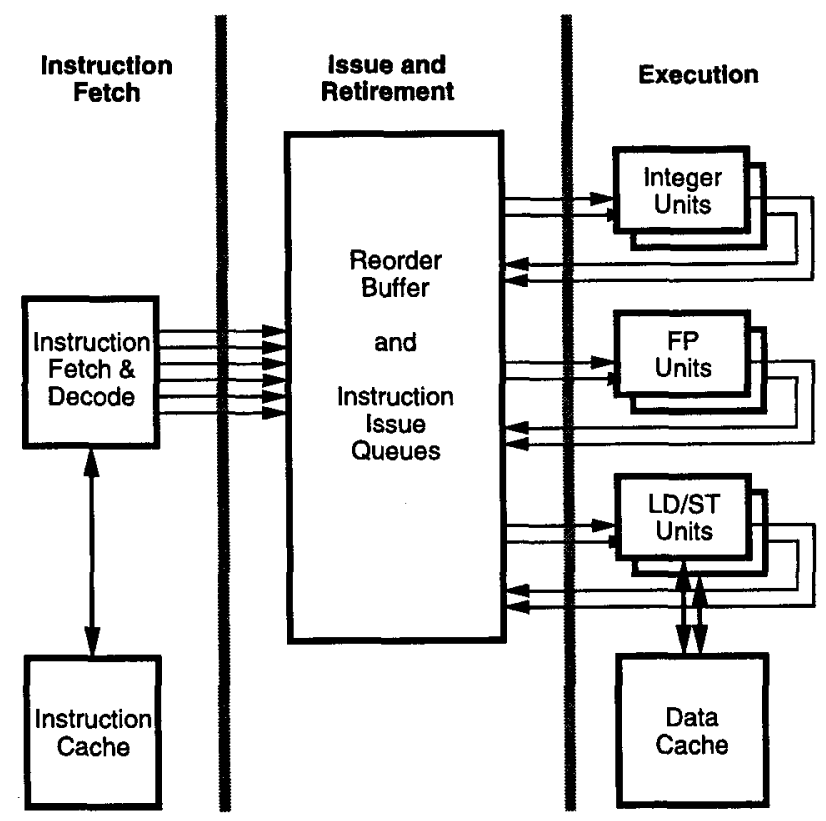

Figure 1. A dynamic superscalar CPU

tures called reservation stations [3]. It is possible to design a dynamically scheduled superscalar microprocessor using reservation stations; Johnson gives a thorough description of this approach [13]. However, the most recent implementations of dynamic superscalar processors have used a structure similar to the one shown in Figure 1. Here register renaming between architectural and physical registers is done explicitly, and instruction scheduling and register dependency tracking between instructions are performed in an instruction issue queue. Examples of microprocessors designed in this manner are the MIPS Technologies R10000 [24] and the HP PA-8000 [14]. In these processors the instruction queue is actually implemented as multiple instruction queues for different classes of instructions (e.g. integer, floating point, load/store). The three major phases of instruction execution in a dynamic superscalar machine are also shown in Figure 1. They are fetch, issue and execute. In the rest of this section we describe these phases and the limitations that will arise in the design of a very wide instruction issue CPU.

The goal of the fetch phase is to present the rest of the CPU with a large and accurate window of decoded instructions. Three factors constrain instruction fetch: mispredicted branches, instruction misalignment, and cache misses. The ability to predict branches correctly is crucial to establishing a large, accurate window of instructions. Fortunately, by using a moderate amount of memory (64Kbit), branch predictors such as the selective branch predictor proposed by McFarling are able to reduce misprediction rates to under 5\% for most programs [15]. However, good branch prediction is not enough. As Conte pointed out, it is also necessary to align a packet of instructions for the decoder [7]. When the issue width is wider than four instructions there is a high probability that it will be necessary to fetch across a branch for a single packet of instructions since, in integer programs, one in every five instructions is a branch [12]. This will require fetching from two cache lines at once and merging the cache lines together to form a single packet of instructions. Conte describes a number of methods for achieving this. A technique that divides the instruction cache into banks and fetches from multiple banks at once is not too expensive to implement and provides performance that is within $3 \%$ of a perfect scheme on an 8-wide issue machine. Even with good branch prediction and alignment a significant cache miss rate will limit the ability of the fetcher to maintain an adequate window of instructions. There are still some applications such as large logic simulations, transactions processing and the OS kernel that have significant instruction cache miss rates even with fairly large $64 \mathrm{~KB}$ two way set-associative caches [19]. Fortunately, it is possible to hide some of the instruction cache miss latency in a dynamically scheduled processor by executing instructions that are already in the instruction window. Rosenblum et. al. have shown that over $60 \%$ of the instruction cache miss latency can be hidden on a database benchmark with a $64 \mathrm{~KB}$ two way set associative instruction cache [19]. Given good branch prediction and instruction alignment it is likely that the fetch phase of a wide-issue dynamic superscalar processor will not limit performance.

In the issue phase, a packet of renamed instructions is inserted into the instruction issue queue. An instruction is issued for execution once all of its operands are ready. There are two ways to implement renaming. One could use an explicit table for mapping architectural registers to physical registers, this scheme is used in the R10000 [24], or one could use a combination reorder buffer/instruction queue as in the PA-8000 [14]. The advantage of the mapping table is that no comparisons are required for register renaming. The disadvantage of the mapping table is that the number of access ports required by the mapping table structure is $O \times W$, where $O$ is the number of operands per instruction and $W$ is the issue width of the machine. An eight-wide issue machine with three operands per instruction requires a 24 port mapping table. Implementing renaming with a reorder buffer has its own set of drawbacks. It requires $n \times Q \times O \times W$ 1-bit comparators to determine which physical registers should supply operands for a new packet of instructions, where $n$ is the number of bits required to encode a register identifier and $Q$ is the size of the instruction issue queue. Clearly, the number of comparators grows with the size of the instruction queue and issue width. Once an instruction is in the instruction queue, all instructions that issue must update their dependencies. This requires another set of $n \times Q \times O \times W$ comparators. For example, a machine with eight wide issue, three operand instructions, a 64entry instruction queue, and 6-bit comparisons requires 9,216 1-bit comparators. The net effect of all the comparison logic and encoding associated with the instruction issue queue is that it takes a large amount of area to implement. On the PA-8000, which is a fourissue machine with 56 instruction issue queue entries, the instruction issue queue takes up $20 \%$ of the die area. In addition, as issue widths increase, larger windows of instructions are required to find independent instructions that can issue in parallel and maintain the full issue bandwidth. The result is a quadratic increase in the size of the instruction issue queue. Moving to the circuit level, the instruction issue queue uses a broadcast mechanism to communicate the tags of the instructions that are issued, which requires wires that span the length of the structure. In future advanced integrated circuit technologies these wires will have increasingly long delays relative to the gates that drive them [9]. Given this situation, ultimately, the instruction issue queue will limit the cycle time of the processor. For these reasons we believe that the instruction issue 
queue will fundamentally limit the performance of wide issue superscalar machines.

In the execution phase, operand values are fetched from the register file or bypassed from earlier instructions to execute on the functional units. The wide superscalar execution model will encounter performance limits in the register file, in the bypass logic and in the functional units. Wider instruction issue requires a larger window of instructions, which implies more register renaming. Not only must the register file be larger to accommodate more renamed registers, but the number of ports required to satisfy the full instruction issue bandwidth also grows with issue width. Again, this causes a quadratic increase in the complexity of the register file with increases in issue width. Farkas et. al. have investigated the effect of register file complexity on performance [10]. They find that an eight-issue machine only performs $20 \%$ better than a four-issue machine when the effect of cycle-time is included in the performance estimates. The complexity of the bypass logic also grows quadratically with number of execution units; however, a more limiting factor is the delay of the wires that interconnect the execution units. As far as the execution units themselves are concerned, the arithmetic functional units can be duplicated to support the issue width, but more ports must be added to the primary data cache to provide the necessary load/store bandwidth. The cheapest way to add ports to the data cache is by building a banked cache [20], but the added multiplexing and control required to implement a banked cache increases the access time of the cache. We investigate this issue in more detail in Section 4.2 .

\section{The Case for a Single-Chip Multiprocessor}

The motivation for building a single chip multiprocessor comes from two sources; there is a technology push and an application pull. We have already argued that technology issues, especially the delay of the complex issue queue and multi-port register files, will limit the performance returns from a wide superscalar execution model. This motivates the need for a decentralized microarchitecture to maintain the performance growth of microprocessors. From the applications perspective, the microarchitecture that works best depends on the amount and characteristics of the parallelism in the applications.

Wall has performed one of the most comprehensive studies of application parallelism [22]. The results of his study indicate that applications fall in two classes. The first class consists of applications with low to moderate amounts of parallelism; under ten instructions per cycle with aggressive branch prediction and large, but not infinite window sizes. Most of these applications are integer applications. The second class consists of applications with large amounts of parallelism, greater than forty instructions per cycle with aggressive branch prediction and large window sizes. The majority of these applications are floating point applications and most of the parallelism is in the form of loop-level parallelism.

The application pull towards a single-chip multiprocessor arises because these two classes of applications require different execution models. Applications in the first class work best on processors that are moderately superscalar ( 2 issue) with very high clock rates because there is little parallelism to exploit. To make this more concrete we note that a $200 \mathrm{MHz}$ MIPS R5000, which is a single issue machine when running integer programs, achieves a SPEC95 inte- ger rating which is $70 \%$ of the rating of a $200 \mathrm{MHz}$ MIPS R 10000 , which is a four-issue machine [6]. Both machines have the same size data and instruction caches, but the R5000 has a blocking data cache, while the R10000 has a non-blocking data cache. Applications in the second class have large amounts of parallelism and see performance benefits from a variety of methods designed to exploit parallelism such as superscalar, VLIW or vector processing. However, the recent advances in parallel compilers make a multiprocessor an efficient and flexible way to exploit the parallelism in these programs [1]. Single-chip multiprocessors, designed so that the individual processors are simple and achieve very high clock rates, will work well on integer programs in the first class. The addition of low latency communication between processors on the same chip also allows the multiprocessor to exploit the parallelism of the floating point programs in the second class. In Section 6 we evaluate the performance of a single-chip multiprocessor for these two application classes.

There are a number of ways to use a multiprocessor. Today, the most common use is to execute multiple processes in parallel to increase throughput in a multiprogramming environment under the control of a multiprocessor aware operating system. We note that there are a number of commercially available operating systems that have this capability (e.g. Silicon Graphics IRIX, Sun Solaris, Microsoft Windows NT). Furthermore, the increasingly widespread use of visualization and multimedia applications tends to increase the number of active processes or independent threads on a desktop machine or server at a particular point in time.

Another way to use a multiprocessor is to execute multiple threads in parallel that come from a single application. Two examples are transaction processing and hand parallelized floating point scientific applications [23]. In this case the threads communicate using shared memory, and these applications are designed to run on parallel machines with communication latencies in the hundreds of CPU clock cycles; therefore, the threads do not communicate in a very fine grained manner. Another example of manually parallelized applications are fine-grained thread-level integer applications. Using the results from Wall's study, these applications exhibit moderate amounts of parallelism when the instruction window size is very large and the branch prediction is perfect because the parallelism that exists is widely distributed. Due to the large window size and the perfect branch prediction it will be very difficult for this parallelism could be extracted with a superscalar execution model. However, it is possible for a programmer that understands the nature of the parallelism in the application to parallelize the application into multiple threads. The parallelism exposed in this manner is fine-grained and cannot be exploited by a conventional multiprocessor architecture. The only way to exploit this type of parallelism is with a single-chip multiprocessor architecture.

A third way to use a multiprocessor is to accelerate the execution of sequential applications without manual intervention; this requires automatic parallelization technology. Recently, this automatic parallelization technology was shown to be effective on scientific applications [2], but it is not yet ready for general purpose integer applications. Like the manually parallelized integer applications, these applications could derive significant performance benefits from the low-latency interprocessor communication provided by a single-chip multiprocessor. 


\begin{tabular}{|c|c|c|}
\hline & 6-way SS & 4x2-way MP \\
\hline \# of CPUs & 1 & 4 \\
\hline Degree superscalar & 6 & $4 \times 2$ \\
\hline \# of architectural registers & $32 \mathrm{int} / 32 \mathrm{fp}$ & $4 \times 32$ int / 32fp \\
\hline \# of physical registers & 160int / 160fp & $4 \times 40 \mathrm{int} / 40 \mathrm{fp}$ \\
\hline \# of integer functional units & 3 & $4 \times 1$ \\
\hline \# of floating pt. functional units & 3 & $4 \times 1$ \\
\hline \# of load/store ports & 8 (one per bank) & $4 \times 1$ \\
\hline BTB size & 2048 entries & $4 \times 512$ entries \\
\hline Return stack size & 32 entries & $4 \times 8$ entries \\
\hline Instruction issue queue size & 128 entries & $4 \times 8$ entries \\
\hline I cache & $32 \mathrm{~KB}, 2$-way S. A. & $4 \times 8 \mathrm{~KB}, 2$-way S. A. \\
\hline D cache & $32 \mathrm{~KB}, 2$-way S. A. & $4 \times 8 \mathrm{~KB}, 2$-way S. A. \\
\hline Ll hit time & 2 cycles $(4 \mathrm{~ns})$ & 1 cycle ( $2 \mathrm{~ns})$ \\
\hline L1 cache interleaving & 8 banks & N/A \\
\hline Unified L2 cache & 256 KB, 2-way S. A. & $256 \mathrm{~KB}, 2$-way S. A. \\
\hline L2 hit time / L1 penalty & 4 cycles ( $8 \mathrm{~ns})$ & 5 cycles $(10 \mathrm{~ns})$ \\
\hline Memory latency / L2 penalty & 50 cycles $(100 \mathrm{~ns})$ & 50 cycles $(100 \mathrm{~ns})$ \\
\hline
\end{tabular}

Table 1. Key characteristics of the two microarchitectures

\section{Two Microarchitectures}

To compare the wide superscalar and multiprocessor design approaches, we have developed the microarchitectures for two machines that will represent the state of the art in processor design a few years from now. The superscalar microarchitecture (SS) is a logical extension of the current R10000 superscalar design, widened from the current four-way issue to a six-way issue implementation. The multiprocessor microarchitecture (MP), is a four-way single-chip multiprocessor composed of four identical 2-way superscalar processors. In order to fit four identical processors on a die of the same size, each individual processor is comparable to the Alpha 21064, which became available in 1992 [8].

These two extremely different microarchitectures have nearly identical die sizes when built in identical process technologies. The processor size we select is based upon the kinds of processor chips that advances in silicon processing technology will allow in the next few years. When manufactured in a $0.25 \mu \mathrm{m}$ process, which should be possible by the end of 1997, each of the chips will have an area of $430 \mathrm{~mm}^{2}$ - about $30 \%$ larger than leading-edge microprocessors being shipped today. This represents typical die size growth over the course of a few years among the largest, fastest microprocessors [11].

We have argued that the simpler two-issue CPU used in the multiprocessor microarchitecture will have a higher clock rate than the six issue CPU; however, for the purposes of this comparison we have assumed that the two processors have the same clock rate. To achieve the same clock rate the wide superscalar architecture would require deeper pipelining due to the large amount of instruction issue logic in the critical path. For simplicity, we ignore latency variations between the architectures due to the degree of pipelining. We assume the clock frequency of both machines is $500 \mathrm{MHz}$. At $500 \mathrm{MHz}$ the main memory latencies experienced by the processor are large. We have modeled the main memory as a 50 -cycle, $100 \mathrm{~ns}$ delay for both architectures, typical values in a workstation today with 60 ns DRAMs and 40 ns of delays due to buffering in the DRAM controller chips [25].

Table 1 shows the key characteristics of the two architectures. We explain and justify these characteristics in the following sections. The integer and floating point functional unit result and repeat latencies are the same as the R10000 [24]

\subsection{6-Way Superscalar Architecture}

The 6-way superscalar architecture is a logical extension of the current R10000 design. As the floorplan in Figure 2 and the area breakdown in Table 2 indicate, the logic necessary for out-of-order instruction issue and scheduling dominates the area of the chip, due to the quadratic area impact of supporting 6-way instruction issue. First, we increased the number of ports in the instruction buffers by $50 \%$ to support 6-way issue instead of 4-way, increasing the area of each buffer by about $30-40 \%$. Second, we increased the number of instruction buffers from 48 to 128 entries so that the processor examines a larger window of instructions for ILP to keep the execution units busy. This large instruction window also compensates for the fact that the simulations do not execute code that is optimized for a 6-way superscalar machine. The larger instruction window size and wider issue width causes a quadratic area increase of the instruction sequencing logic to 3-4 times its original size. Altogether, the logic necessary to handle out-of-order instruction issue occupies about $120 \mathrm{~mm}^{2}$ - about $30 \%$ of the die. In comparison, the actual execution units only occupy about $70 \mathrm{~mm}^{2}-$ just $18 \%$ of the die is required to build triple R 10000 execution units in a $0.25 \mu \mathrm{m}$ process.

Due to the increased rate at which instructions are issued, we also enhanced the fetch logic by increasing the size of the branch target buffer to 2048 entries and the call-return stack to 32 entries. This increases the branch prediction accuracy of the processor and pre- 


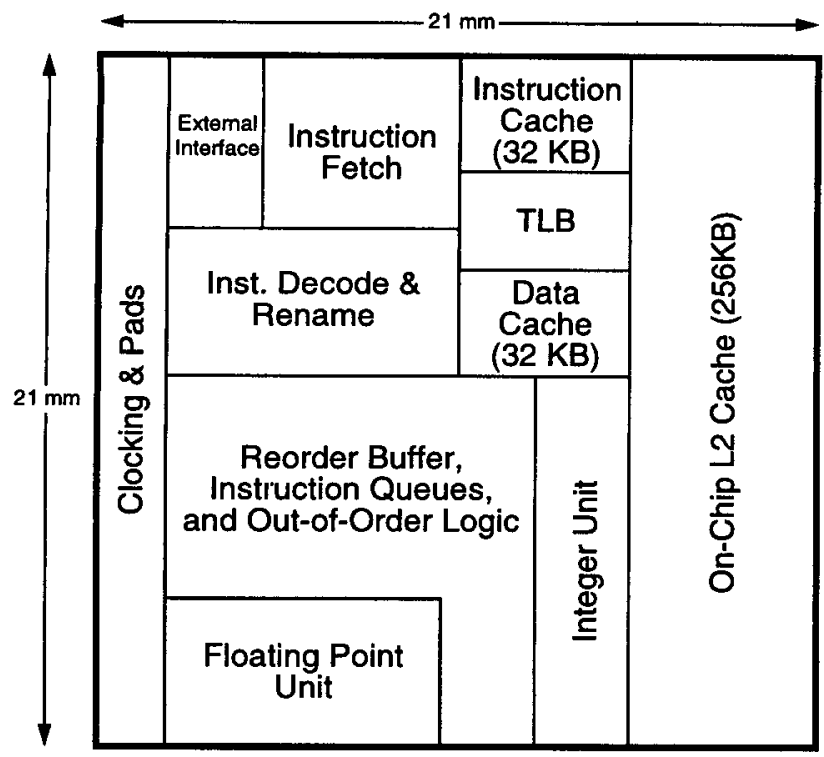

Figure 2. Floorplan for the six-issue dynamic superscalar microprocessor.

vents the instruction fetch mechanism from becoming a bottleneck since the 6-way execution engine requires a much higher instruction fetch bandwidth than the 2-way processors used in the MP architecture.

The on-chip memory hierarchy is similar to the Alpha 21164 - a small, fast level one (L1) cache backed up by a large on-chip level two (L2) cache. The wide issue width requires the L1 cache to support wide instruction fetches from the instruction cache and multiple loads from the data cache during each cycle. The two-way set associative $32 \mathrm{~KB} \mathrm{L1}$ data cache is banked eight ways into eight small, single-ported, independent $4 \mathrm{~KB}$ cache banks each of which handling one access every 2 ns processor cycle. However, the additional overhead of the bank control logic and crossbar required to arbitrate between the multiple requests sharing the 8 data cache banks adds another cycle to the latency of the $\mathrm{L} 1$ cache, and increases the area by $25 \%$. Therefore, our modeled $\mathrm{L} 1$ cache has a hit time of 2 cycles. Backing up the $32 \mathrm{~KB} \mathrm{L1}$ caches is a large, unified, $256 \mathrm{~KB} \mathrm{~L} 2$ cache that takes 4 cycles to access. These latencies are simple extensions of the times obtained for the $\mathrm{L} 1$ caches of current Alpha microprocessors [4], using a $0.25 \mu \mathrm{m}$ process technology

\subsection{4 x 2-way Superscalar Multiprocessor Architecture}

The MP architecture is made up of four 2-way superscalar processors interconnected by a crossbar that allows the processors to share the L2 cache. On the die, the four processors are arranged in a grid with the L2 cache at one end, as shown in Figure 3. Internally, each of the processors has a register renaming buffer that is much more limited than the one in the 6-way architecture, since each CPU only has an 8-entry instruction buffer. We also quartered the size of the branch prediction mechanisms in the fetch units, to 512 BTB entries and 8 call-return stack entries. After the area adjustments caused by these factors are accounted for, each of the four proces-

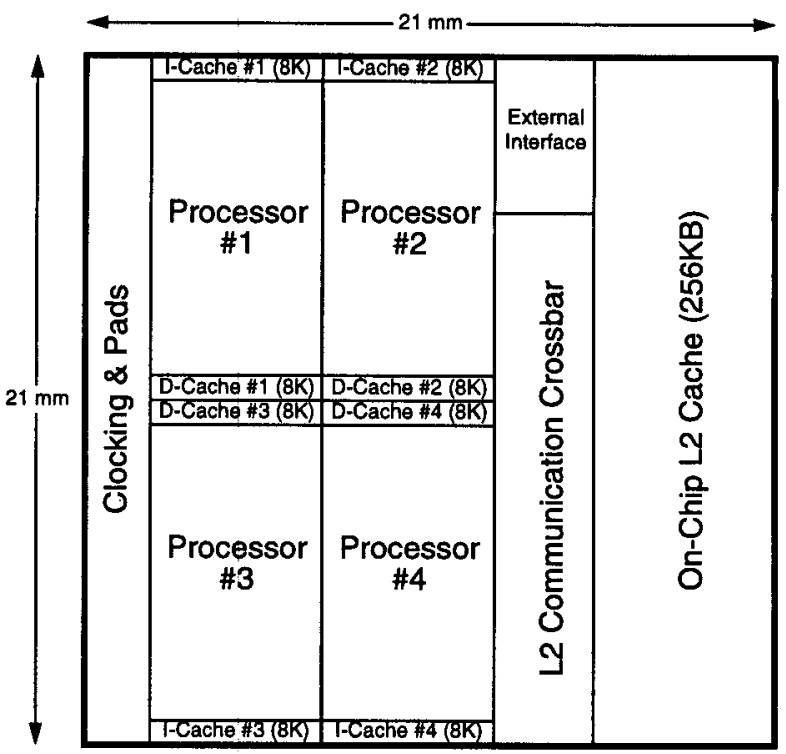

Figure 3. Floorplan for the four-way single-chip multiprocessor.

sors is less than one-fourth the size of the 6-way SS processor, as shown in Table 3. The number of execution units actually increases in the MP because the 6-way processor had three units of each type, while the 4-way MP must have four - one for each CPU. On the other hand, the issue logic becomes dramatically smailer, due to the decrease in instruction buffer ports and the smaller number of entries in each instruction buffer. The scaling factors of these two units balance each other out, leaving the entire processor very close to one-fourth of the size of the 6-way processor.

The on-chip cache hierarchy of the multiprocessor is significantly different from the cache hierarchy of the 6-way superscalar processor. Each of the 4 processors has its own single-banked and singleported $8 \mathrm{~KB}$ instruction and data caches that can both be accessed in a single 2 ns cycle. Since each cache can only be accessed by a single processor with a single load/store unit, no additional overhead is incurred to handle arbitration among independent memoryaccess units. However, since the four processors now share a single L2 cache, that cache requires an extra cycle of latency during every access to allow time for interprocessor arbitration and crossbar delay. We model this additional L2 delay by penalizing the MP an additional cycle on every L2 cache access, resulting in a 5 cycle L2 hit time.

\section{Simulation Methodology}

Accurately evaluating the performance of the two microarchitectures requires a way of simulating the environment in which we would expect these architectures to be used in real systems. In this section we describe the simulation environment and the applications used in this study.

\subsection{Simulation Environment}

We execute the applications in the SimOS simulation environment [18]. SimOS models the CPUs, memory hierarchy and I/O devices 


\begin{tabular}{|c|c|c|c|c|c|}
\hline CPU Component & $\begin{array}{l}0.35 \mu \mathrm{m} \text { R10K } \\
\text { Original Size }\left(\mathrm{mm}^{2}\right)\end{array}$ & $\begin{array}{l}\text { Size Extrapolated } \\
\text { to } 0.25 \mu \mathrm{m}\left(\mathrm{mm}^{2}\right)\end{array}$ & $\begin{array}{l}\text { \% Growth Due to } \\
\text { New Functionality }\end{array}$ & New Size $\left(\mathrm{mm}^{2}\right)$ & $\%$ Area \\
\hline 256K On-Chip L2 Cache ${ }^{a}$ & 219 & 112 & $0 \%$ & 112 & $26 \%$ \\
\hline 8-bank D Cache (32 KB) & 26 & 13 & $25 \%$ & 17 & $4 \%$ \\
\hline 8-bank I Cache (32 KB) & 28 & 14 & $25 \%$ & 18 & $4 \%$ \\
\hline TLB Mechanism & 10 & 5 & $200 \%$ & 15 & $3 \%$ \\
\hline External Interface Unit & 27 & 14 & $0 \%$ & 14 & $3 \%$ \\
\hline Instruction Fetch Unit and BTB & 18 & 9 & $200 \%$ & 28 & $6 \%$ \\
\hline Instruction Decode Section & 21 & 11 & $250 \%$ & 38 & $9 \%$ \\
\hline Instruction Queues & 28 & 14 & $250 \%$ & 50 & $12 \%$ \\
\hline Reorder Buffer & 17 & 9 & $300 \%$ & 34 & $9 \%$ \\
\hline Integer Functional Units & 20 & 10 & $200 \%$ & 31 & $7 \%$ \\
\hline FP Functional Units & 24 & 12 & $200 \%$ & 37 & $9 \%$ \\
\hline Clocking \& Overhead & 73 & 37 & $0 \%$ & 37 & $9 \%$ \\
\hline Total Size & - & - & - & 430 & $100 \%$ \\
\hline
\end{tabular}

Table 2. Size extrapolations for the 6-way superscalar from the MIPS R10000 processor

\begin{tabular}{|c|c|c|c|c|c|}
\hline CPU Component & $\begin{array}{l}0.35 \mu \mathrm{m} \text { R10K } \\
\text { Original Size }\left(\mathrm{mm}^{2}\right)\end{array}$ & $\begin{array}{l}\text { Size Extrapolated } \\
\text { to } 0.25 \mu \mathrm{m}\left(\mathrm{mm}^{2}\right)\end{array}$ & $\begin{array}{l}\% \text { Growth Due to } \\
\text { New Functionality }\end{array}$ & New Size $\left(\mathrm{mm}^{2}\right)$ & $\begin{array}{l}\% \text { Area } \\
\text { (of CPU / of entire } \\
\text { chip) }\end{array}$ \\
\hline D Cache $(8 \mathrm{~KB})$ & 26 & 13 & $-75 \%$ & 3 & $6 \% / 3 \%$ \\
\hline I Cache (8 KB) & 28 & 14 & $-75 \%$ & 4 & $7 \% / 3 \%$ \\
\hline TLB Mechanism & 10 & 5 & $0 \%$ & 5 & $9 \% / 5 \%$ \\
\hline Instruction Fetch Unit and BTB & 18 & 9 & $-25 \%$ & 7 & $13 \% / 7 \%$ \\
\hline Instruction Decode Section & 21 & 11 & $-50 \%$ & 5 & $10 \% / 5 \%$ \\
\hline Instruction Queues & 28 & 14 & $-70 \%$ & 4 & $8 \% / 4 \%$ \\
\hline Reorder Buffer & 17 & 9 & $-80 \%$ & 2 & $3 \% / 2 \%$ \\
\hline Integer Functional Units & 20 & 10 & $0 \%$ & 10 & $20 \% / 10 \%$ \\
\hline FP Functional Units & 24 & 12 & $0 \%$ & 12 & $23 \% / 12 \%$ \\
\hline Per-CPU Subtotal & - & - & - & 53 & $100 \% / 50 \%$ \\
\hline 256K On-Chip L2 Cache ${ }^{2}$ & 219 & 112 & $0 \%$ & 112 & $26 \%$ \\
\hline External Interface Unit & 27 & 14 & $0 \%$ & 14 & $3 \%$ \\
\hline Crossbar Between CPUs & - & - & - & 50 & $12 \%$ \\
\hline Clocking \& Overhead & 73 & 37 & $0 \%$ & 37 & $9 \%$ \\
\hline Total Size & - & - & - & 424 & $100 \%$ \\
\hline
\end{tabular}

Table 3. Size extrapolations in the $4 \times 2$-way MP from the MIPS R10000 processor.

a. estimated from current $\mathrm{Ll}$ caches

of uniprocessor and multiprocessor systems in sufficient detail to boot and run a commercial operating system. SimOS uses the MIPS-2 instruction set and runs the Silicon Graphics IRIX 5.3 operating system which has been tuned for multiprocessor performance. SimOS actually simulates the operating system; therefore, all the memory references made by the operating system and the applications are generated. This feature is particularly important for the study of multiprogramming workloads where the time spent executing kernel code makes up a significant fraction of the nonidle execution time.

A unique feature of SimOS that makes studies such as this feasible is that SimOS supports multiple CPU simulators that use a common instruction set architecture. This allows trade-offs to be made between the simulation speed and accuracy. The fastest CPU simu- lator, called Embra, uses binary-to-binary translation techniques and is used for booting the operating system and positioning the workload so that we can focus on interesting regions of execution. The medium performance CPU simulator, called Mipsy, is two orders of magnitude slower than Embra. Mipsy is an instruction set simulator that models all instructions with a one cycle result latency and a one cycle repeat rate. Mipsy interprets all user and privileged instructions and feeds memory references to a memory system simulator. The slowest, most detailed CPU simulator is MXS, which supports dynamic scheduling, speculative execution and non-blocking memory references. MXS is over four orders of magnitude slower than Embra.

The cache and memory system component of our simulator is completely event-driven and interfaces to the SimOS processor model 


\begin{tabular}{|l|l|}
\hline \multicolumn{2}{|c|}{ Integer applications } \\
\hline \hline compress & compresses and uncompresses file in memory \\
\hline eqntott & translates logic equations into truth tables \\
\hline m88ksim & Motorola 88000 CPU simulator \\
\hline MPsim & VCS compiled Verilog simulation of a multiprocessor \\
\hline \multicolumn{2}{|c|}{ Floating point applications } \\
\hline \hline applu & solver for parabolic/elliptic partial differential equations \\
\hline apsi & solves problems of temperature, wind, velocity, and distribution of pollutants \\
\hline swim & shallow water model with 1K $\times 1 \mathrm{~K}$ grid \\
\hline tomcatv & mesh-generation with Thompson solver \\
\hline \multicolumn{2}{|c|}{ Multiprogramming application } \\
\hline \hline pmake & parallel make of gnuchess using C compiler \\
\hline
\end{tabular}

Table 4. The applications.

which drives it. Processor memory references cause threads to be generated which keep track of the state of each memory reference and the resource usage in the memory system. A call-back mechanism is used to inform the processor of the status of all outstanding references, and to inform the processor when a reference completes. These mechanisms allow for very detailed cache and memory system models, which include cycle accurate measures of contention and resource usage throughout the system.

\subsection{Applications}

The performance of nine realistic applications is used to evaluate the two microarchitectures. Table 4 shows that the nine applications are made up of two SPEC95 integer benchmarks (compress, m88ksim), one SPEC92 integer benchmark (eqntott), one other integer application (MPsim), four SPEC95 floating point benchmarks (applu, apsi, swim, tomcatv), and a multiprogramming application (pmake).

The applications are parallelized in different ways to run on the MP microarchitecture. Compress is run unmodified on both the SS and MP microarchitectures; using only one processor of the MP architecture. Eqntott is parallelized manually by modifying a single bit vector comparison routine that is responsible for $90 \%$ of the execution time of the application [16]. The CPU simulator m88ksim is also parallelized manually into three threads using the SUIF compiler runtime system. Each of the three threads is allowed to be in a different phase of simulating a different instruction at the same time. This style of parallelization is very similar to the overlap of instruction execution that occurs in hardware pipelining. The MPsim application is a Verilog model of a bus based multiprocessor running under a multi-threaded compiled code simulator (Chronologic VCS-MT). The multiple threads are specified manually by assigning parts of the model hierarchy to different threads. The MPsim application uses four closely coupled threads; one for each of the processors in the model. The parallel versions of the SPEC95 floating point benchmarks are automatically generated by the SUIF compiler system [2]. The pmake application is a program development workload that consists of the compile phase of the Modified Andrew Benchmark [17]. The same pmake application is executed on both microarchitectures; however, the OS takes advantage of the extra processors in the MP microarchitecture to run multiple compilations in parallel.

A difficult problem that arises when comparing the performance of different processors is ensuring that they do the same amount of work. The solution is not as easy as comparing the execution times of each application on each machine. Due to the slow simulation speed of the detailed CPU simulator (MXS) used to collect these results it would take far too long to run the applications to completion. Our solution is to compare the two microarchitectures over a portion of the application using a technique called representative execution windows [5]. In most compute intensive applications there is a steady state execution region that consists of a single outer loop or a set of loops that makes up the bulk of the execution time. It is sufficient to sample a small number of iterations of these loops as a representative execution window if the execution time behavior of the window is indeed representative of the entire program. Simulation results show that for most applications the cache miss rates and the number of instructions executed in the window deviates by less than $1 \%$ from the results for the entire program.

The simulation procedure begins with a checkpoint taken with the Embra simulator. Simulation from the checkpoint starts with the instruction level simulator Mipsy and the full memory system. After the caches are warmed by running the Mipsy simulator through the representative execution window at least once, the simulator is switched to the detailed simulator, MXS, to collect the performance results presented in this paper.

We use the technique of representative execution windows for all the applications except pmake. Pmake does not have a well defined execution region that is representative of the application as a whole. Therefore, the results for pmake are collected by running the entire application with MXS.

\section{Performance Comparison}

We begin by examining the performance of the superscalar microarchitecture and one processor of the multiprocessor microarchitecture. Table 5 shows the IPC, branch prediction rates and cache miss rates for one processor of the MP; Table 6 shows the IPC, branch prediction rates, and cache miss rates for the SS 
microarchitecture. The cache miss rates are presented in the tables in terms of misses per completed instruction (MPCI); including instructions that complete in kernel and user mode. When the issue width is increased from two to six we see that the actual IPC increases by less than a factor of 1.6 for all of the integer and multiprogramming applications. For the floating point applications the performance improvement varies from a factor of 1.6 for tomcatv to 2.4 for swim.

\begin{tabular}{|l|c|c|c|c|c|}
\hline Program & IPC & $\begin{array}{c}\text { BP Rate } \\
\text { \% }\end{array}$ & $\begin{array}{c}\text { I cache } \\
\text { \% MPCI }\end{array}$ & $\begin{array}{c}\text { D cache } \\
\text { \%MPCI }\end{array}$ & $\begin{array}{c}\text { L2 cache } \\
\text { \%MPCI }\end{array}$ \\
\hline \hline compress & 0.9 & 85.9 & 0.0 & 3.5 & 1.0 \\
\hline eqntott & 1.3 & 79.8 & 0.0 & 0.8 & 0.7 \\
\hline m88ksim & 1.4 & 91.7 & 2.2 & 0.4 & 0.0 \\
\hline MPsim & 0.8 & 78.7 & 5.1 & 2.3 & 2.3 \\
\hline applu & 0.9 & 79.2 & 0.0 & 2.0 & 1.7 \\
\hline apsi & 0.6 & 95.1 & 1.0 & 4.1 & 2.1 \\
\hline swim & 0.9 & 99.7 & 0.0 & 1.2 & 1.2 \\
\hline tomcatv & 0.8 & 99.6 & 0.0 & 7.7 & 2.2 \\
\hline pmake & 1.0 & 86.2 & 2.3 & 2.1 & 0.4 \\
\hline
\end{tabular}

Table 5. Performance of a single 2-issue superscalar processor.

\begin{tabular}{|l|c|c|c|c|c|}
\hline Program & IPC & $\begin{array}{c}\text { BP Rate } \\
\text { \% }\end{array}$ & $\begin{array}{c}\text { I cache } \\
\text { \%MPCI }\end{array}$ & $\begin{array}{c}\text { D cache } \\
\text { \%MPCI }\end{array}$ & $\begin{array}{c}\text { L2 cache } \\
\text { \%MPCI }\end{array}$ \\
\hline \hline compress & 1.2 & 86.4 & 0.0 & 3.9 & 1.1 \\
\hline eqniott & 1.8 & 80.0 & 0.0 & 1.1 & 1.1 \\
\hline m88ksim & 2.3 & 92.6 & 0.1 & 0.0 & 0.0 \\
\hline MPsim & 1.2 & 81.6 & 3.4 & 1.7 & 2.3 \\
\hline applu & 1.7 & 79.7 & 0.0 & 2.8 & 2.8 \\
\hline apsi & 1.2 & 95.6 & 0.2 & 3.1 & 2.6 \\
\hline swim & 2.2 & 99.8 & 0.0 & 2.3 & 2.5 \\
\hline tomcatv & 1.3 & 99.7 & 0.0 & 4.2 & 4.3 \\
\hline pmake & 1.4 & 82.7 & 0.7 & 1.0 & 0.6 \\
\hline
\end{tabular}

Table 6. Performance of the 6-issue superscalar processor.

One of the major causes of processor stalls in a superscalar processor is cache misses. However, cache misses in a dynamically scheduled superscalar processor with speculative execution and nonblocking caches are not straightforward to characterize. The cache misses that occur in a single issue in-order processor are not necessarily the same as the misses that will occur in the speculative outof-order processor. In speculative processors there are misses that are caused by speculative instructions that never complete. With non-blocking caches, misses may also occur to lines which already have outstanding misses. Both types of misses tend to inflate the cache miss rate of a speculative out-of-order processor. The second type of miss is mainly responsible for the higher $\mathrm{L} 2$ cache miss rates of the 6-issue processor compared to the 2-issue processor, even though the cache sizes are equal.

Figure 4 shows the IPC breakdown for one processor of the MP microarchitecture with an ideal IPC of two. In addition to the actual IPC achieved, we show the loss in IPC due to data and instruction cache stalls, and pipeline stalls. We see that a large percentage of the IPC loss is due to data cache stall time. This is caused by the small size of the primary data cache. Mk88ksim, MPsim and pmake have significant instruction cache stall time which is due to the large instruction working set size of these applications. Pmake also has multiple processes and significant kernel execution time which further increases the instruction cache miss rate.

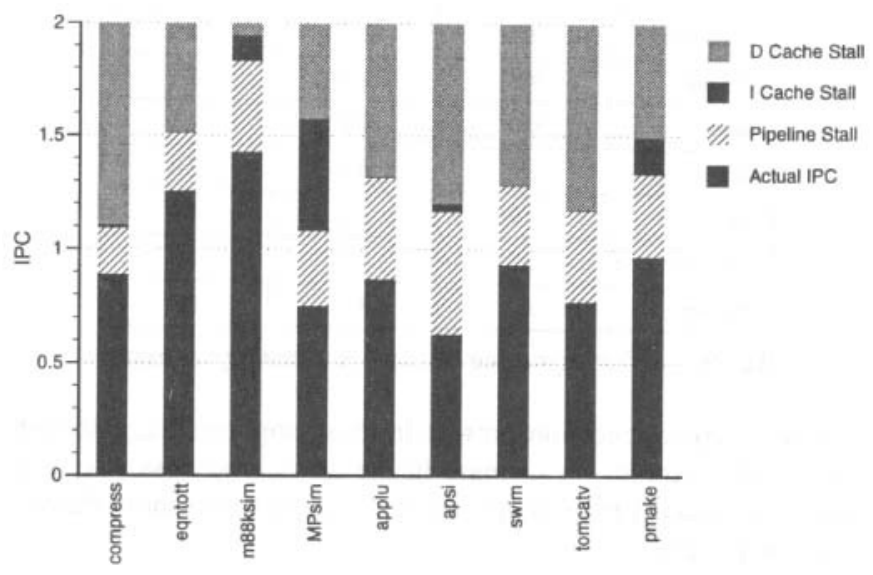

Figure 4. IPC Breakdown for a single 2-issue processor.

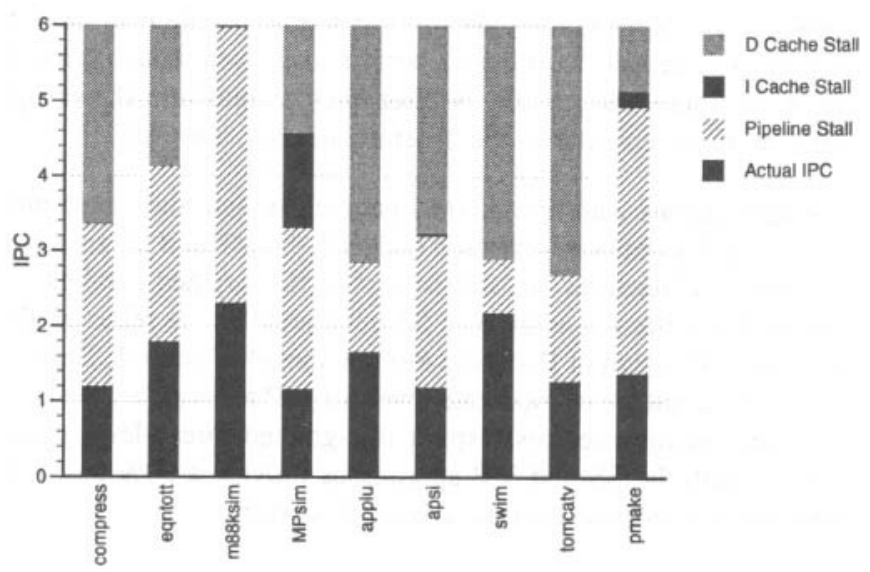

Figure 5. IPC Breakdown for the 6-issue processor.

Figure 5 shows the IPC breakdown for the SS microarchitecture. We see that a significant amount of IPC.is lost due to pipeline stalls. The increase in pipeline stalls relative to the two-issue processor is due to limited ILP in the applications and the 2-cycle L1 data cache hit time. The larger instruction cache in the SS microarchitecture eliminates most of the stalls due to instruction misses for all of the applications except MPsim and pmake. Although the SPEC95 floating point applications have a significant amount of ILP, their performance is limited on the SS microarchitecture due to data cache stalls which consume over one-half of the available IPC

Table 7 shows cache miss rates for the MP microarchitecture given in terms of MPCI. To reduce miss-rate effects caused by the idle loop and spinning due to synchronization, the number of completed instructions are those of the single 2-issue processor. Comparing Table 5 and Table 7 shows that for eqntott, m88ksim and apsi the MP microarchitecture has significantly higher data cache miss rates than the single 2 -issue processor. This is due primarily to the high- 


\begin{tabular}{|l|c|c|c|}
\hline Application & $\begin{array}{c}\text { I cache } \\
\text { \% MPCI }\end{array}$ & $\begin{array}{c}\text { D cache } \\
\text { \% MPCI }\end{array}$ & $\begin{array}{c}\text { L2 cache } \\
\text { \% MPCI }\end{array}$ \\
\hline \hline compress & 0.0 & 3.5 & 1.0 \\
\hline eqntott & 0.6 & 5.4 & 1.2 \\
\hline m88ksim & 2.3 & 3.3 & 0.0 \\
\hline MPsim & 4.8 & 2.5 & 3.4 \\
\hline applu & 0.0 & 2.1 & 1.8 \\
\hline apsi & 2.7 & 6.9 & 2.0 \\
\hline swim & 0.0 & 1.2 & 1.5 \\
\hline tomcatv & 0.0 & 7.8 & 2.5 \\
\hline pmake & 2.4 & 4.6 & 0.7 \\
\hline
\end{tabular}

Table 7. Performance of the $4 \times 2$-issue processor.

degree of communication present in these applications. Although pmake also exhibits an increase in the data cache miss rate, it is caused by process migration from processor to processor in the MP microarchitecture.

Figure 6 shows the performance comparison between the SS and MP microarchitectures. The performance is measured as the speedup of each microarchitecture relative to the single 2-issue processor. On compress, an application with little parallelism, the MP is able to achieve $75 \%$ of the SS performance even though three of the four processors are idle. Neither microarchitecture shows significant improvement over the 2 -issue processor, however.

For applications with fine-grained parallelism and high-communication, such as eqntott, $\mathrm{m} 88 \mathrm{ksim}$ and apsi, the MP and SS are similar. Both architectures are able to exploit fine-grained parallelism, although in different ways. The SS microarchitecture relies on the dynamic extraction of ILP from a single thread of control. The MP can take advantage of moderate levels of ILP and can, unlike conventional multiprocessors, exploit fine-grained thread-level parallelism. Both the SS and MP approaches provide a $30 \%$ to $100 \%$ performance increase over the 2 -issue processor.

Applications with large amounts of parallelism allow the MP microarchitecture to take advantage of coarse-grained parallelism in addition to fine-grained parallelism and ILP. For these applications, the MP is able to significantly outperform the SS microarchitecture, whose ability to dynamically extract parallelism is limited by the 128 instruction window.

\section{Conclusions}

The characteristics of advanced integrated circuit technologies require us to look for new ways to utilize large numbers of gates and mitigate the effects of high interconnect delays. We have discussed the details of implementing both a wide, dynamically scheduled superscalar processor and a single chip multiprocessor. The implementation complexity of the dynamic issue mechanisms and size of the register files scales quadratically with increasing issue width and ultimately impacts the cycle time of the machine. The alternative multiprocessor microarchitecture, which is composed of simpler processors, can be implemented in approximately the same area. We believe that the multiprocessor microarchitecture will be easier to implement and will reach a higher clock rate.

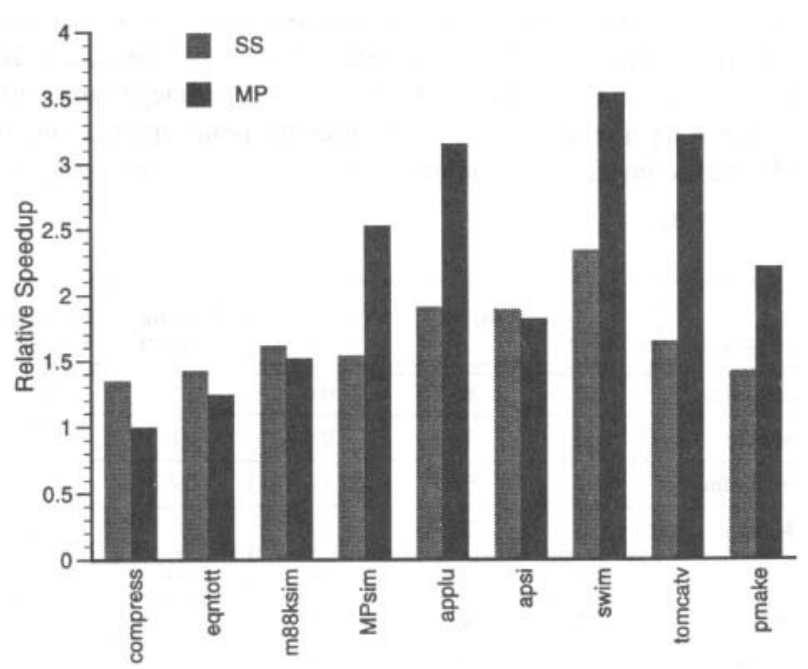

Figure 6. Performance comparison of SS and MP.

Our results show that on applications that cannot be parallelized the superscalar microarchitecture performs $30 \%$ better than one processor of the multiprocessor architecture. On applications with fine grained thread-level parallelism the multiprocessor microarchitecture can exploit this parallelism so that the superscalar microarchitecture is at most $10 \%$ better, even at the same clock rate. We anticipate that the higher clock rates possible with simpler CPUs in the multiprocessor will eliminate this small performance difference. On applications with large grained thread-level parallelism and multiprogramming workloads the multiprocessor microarchitecture performs $50-100 \%$ better than the wide superscalar microarchitecture.

\section{Acknowledgments}

We would like to thank Edouard Bugnion, Mendel Rosenblum, Ben Verghese and Steve Herrod for their help with SimOS, Doug Williams for his assistance with MXS, the SUIF compiler group for use of their applications, and the reviewers for their insightful comments. This work was supported by DARPA contracts DABT63-95C-0089 and DABT63-94-C-0054. 


\section{References}

[1] S. P. Amarasinghe, J. M. Anderson, M. S. Lam, and C.-W. Tseng, "An overview of the SUIF compiler for scalable parallel machines," Proceedings of the Seventh SIAM Conference on Parallel Processing for Scientific Compiler, San Francisco, 1995.

[2] S. Amarasinghe et.al., "Hot compilers for future hot chips," presented at Hot Chips VII, Stanford, CA, 1995.

[3] D. W. Anderson, F. J. Sparacio, and R. M. Tomasulo, "The IBM System $/ 360$ model 91: Machine philosophy and instruction-handling," IBM Journal of Research and Development, vol. 11, pp. 8-24, 1967.

[4] W. Bowhill et. al., "A 300MHz 64b quad-issue CMOS microprocessor," IEEE International Solid-State Circuits Conference Digest of Technical Papers, pp. 182-183, San Francisco, CA, 1995.

[5] E. Bugnion, J. Anderson, T. Mowry, M. Rosenblum, and M. Lam. "Compiler-Directed Page Coloring for Multiprocessors," Proceedings Seventh International Symp. Architectural Support for Programming Languages and Operating Systems (ASPLOS VII), October 1996.

[6] “Chart watch: RISC processors," Microprocessor Report, vol. 10, no. 1, p. 22, January, 1996.

[7] T. Conte, K. Menezes, P. Mills, and B. Patel, "Optimization of instruction fetch mechanisms for high issue rates," Proceedings of the 22nd Annual International Symposium on Computer Architecture, pp. 333-344, Santa Margherita Ligure, Italy, June, 1996.

[8] D. Dobberpuhl et. al., "A 200-MHz 64-b dual-issue CMOS microprocessor," IEEE Journal of Solid-State Circuits, vol. 27, pp. 1555-1557, 1992.

[9] Don Drappper, "The interconnect nightmare," IEEE International Solid-State Circuits Conference Digest of Technical Papers, p. 278, San Francisco, CA, 1996.

[10] K. Farkas, N. Jouppi, and P. Chow, "Register file considerations in dynamically scheduled processors," Proceedings of the 2nd Int. Symp. on High-Performance Computer Architecture, pp. 40-51, San Jose, CA, February, 1996.

[11] J. Hennessy and N. Jouppi, "Computer technology and architecture: an evolving interaction," IEEE Computer Magazine, vol. 24, no. 1, pp. 18-29, 1991.

[12] J. L. Hennessy and D. A. Patterson, Computer Architecture A Quantitative Approach 2nd Edition. San Francisco, California: Morgan Kaufman Publishers, Inc., 1996.

[13] M. Johnson, Superscalar Microprocessor Design. Englewood Cliffs, NJ: Prentice Hall, Inc., 1991

[14] J. Lotz, G. Lesartre, S. Naffzinger, and D. Kipp, "A quad issue out-of-order RISC CPU,"IEEE International Solid-State Circuits Conference Digest of Technical Papers, pp. 210 211, San Francisco, CA, 1996.

[15] S. McFarling, "Combining branch predictors," WRL Technical Note TN-36, Digital Equipment Corporation, 1993.

[16] B. A. Nayfeh, L. Hammond, and K. Olukotun, "Evaluating alternatives for a multiprocessor microprocessor," Proceedings of 23rd Int. Symp. Computer Architecture, pp. 66-77, Philadelphia, PA, 1996.

[17] J. Ousterhout, "Why aren't operating systems getting faster as fast as hardware?," Summer 1990 USENIX Conference, pp. 247-256, June 1990.

[18] M. Rosenblum, S. Herrod, E. Witchel, and A. Gupta, "The
SimOS approach" IEEE Parallel and Distributed Technology, vol. 4, no. 3, 1995.

[19] M. Rosenblum, E. Bugnion, S. Herrod, E. Witchel, and A. Gupta, "The impact of architectural trends on operating system performance," Proceedings of 15th ACM symposium on Operating Systems Principles, Colorado, December, 1995

[20] G. Sohi and M. Franklin, "High Bandwidth Data Memory Systems for Superscalar Processors," Proceedings of 4th Int. Conf. Architectural Support for Programming Languages and Operating Systems (ASPLOS-IV), pp. 5362, April, 1991.

[21] J. E. Thornton, "Parallel operation in the Control Data 6600," Proceedings of Spring Joint Computer Conference, 1964.

[22] D. W. Wall, "Limits of Instruction-Level Parallelism," Digital Western Research Laboratory, WRL Research Report 93/ 6, November 1993.

[23] S. C. Woo, M. Ohara, E. Torrie, J.P. Singh and A. Gupta, "The SPLASH-2 Programs: Characterization and Methodological Considerations", 22nd Annual Int. Symp. Computer Architecture, Santa Margherita, Italy, June 1995.

[24] K. Yeager et. al., "R10000 Superscalar Microprocessor," presented at Hot Chips VII, Stanford, CA, 1995.

[25] J. Zurawski, J. Murray and P. Lemmon, "The design and verification of the AlphaStation 600 5-series workstation," Digital Technical Journal, vol. 7, no. 1, pp. 89-99, 1995. 